\title{
Societal impact of Sustainable Computing
}

\author{
Riddhi Bhardwaj ${ }^{1}$, Faraz Doja ${ }^{2}$ \\ \{riddhi1500@gmail.com ${ }^{1}$, farazdoja91@gmail.com ${ }^{2}$ \} \\ Department of Computer Science, Jamia Hamdard, Block D, Hamdard Nagar, New Delhi
}

\begin{abstract}
Sustainable Computing is the way to provide sustainability considering all aspects like software, hardware as well as the energy consumption process. This paper discusses the need of sustainable computing and the various problems that can and are cured by sustainable computing through Open Data, Big Data and Artificial Intelligence. Since everything in this universe has its pros and cons, so do the above mentioned solutions, which are also discussed in the paper. The paper concludes by describing how sustainable computing has benefitted us in the past and will do the same in the future.
\end{abstract}

Keywords: sustainability, computer systems, hardware, software, consumption process, Big Data, Open Data, Artificial Intelligence

\section{Introduction}

Sustainable development is the idea of development that accommodates the needs of the present needs without risking the requirement of the future generations. The main focus behind this is to maintain a balance between the social, economic and ecological factors so as to achieve a sustainable future. It is inspired from branches like information technology and statistics for creating environmental and economical balance[1]. It comprises of various methods, policies and actions that use information technology whenever and however possible. It is an approach considering total cost of ownership, the total environmental impact and the total benefit of technology systems.

\section{Need of Sustainable Computing}

Recent growth in technology and development of Information and Communication Technology (ICT) devices and software's have impacted the environment by creating issues of energy consumption and heat dissipation[2]. Hence, sustainable computing has been moved to a higher priority. It represents the way for reduction of power requirement in computing infrastructures, limiting energy consumption from renewable energy sources in the environment. It is proved statistically that the rate at which ICT devices are being produced is proportional to the increase in the energy consumption and heat dissipation by these devices [2]. It is now a fact that the world is becoming more and more dependent on ICT and personal computers (PCs) see Table 1 . 
Table1. IDC Forecast of sales of PCs and Laptops. (Source: 'Cloud Computing and Consumer Electronics: A Perfect Match or a Hidden Storm?’ by Peter Corcoran)[3]

\section{IDC Forecast of sales of PCs and Laptops}

\begin{tabular}{|c|c|c|c|c|}
\hline Year & $\begin{array}{l}\text { PC } \\
\text { (Mature) }\end{array}$ & $\begin{array}{l}\text { Laptop } \\
\text { (Mature) }\end{array}$ & $\begin{array}{l}\text { PC } \\
\text { (Developer) }\end{array}$ & $\begin{array}{l}\text { Laptop } \\
\text { (Develepor) }\end{array}$ \\
\hline 2010 & 57.7 & 108.8 & 88.1 & 92.3 \\
\hline 2011 & 54.9 & 105.4 & 92.1 & 109.2 \\
\hline 2012 & 56 & 116.7 & 96.3 & 129.5 \\
\hline 2013 & 54.5 & 127.3 & 100 & 156.7 \\
\hline 2014 & 53.4 & 141.3 & 102.6 & 190.6 \\
\hline 2015 & 59.3 & 153.6 & 104.9 & 229.7 \\
\hline
\end{tabular}

The above table depicts the worldwide growth rate of sales of Servers, Desktop PCs and Mobiles up to the year of 2015[3]. It is observed that sale of Mobile PCs is continuously gearing up whereas there is a slowdown in use of Desktop PCs from year 2012 onwards. Since, ICT comprises of laptops and PCs, they are the main reason for the immense amount of power consumption. As a result many companies have increased their demand for power production, since they feel that their present power is not adequate to meet the ever growing demands of consumers. To solve this problem the idea of sustainable computing came into vision whose main concern was to reduce the usage of power consumption by ICT devices. The concept of Sustainable computing is increasing in popularity. The three major aspects of sustainable computing are:

1. Reduction of energy consumption from any running process on the system.

2. Ensuring longer life cycle of any computing equipment.

3. Ensuring that the need of energy is met from the energy available from all resources in the environment.

Sustainable development goals could be achieved by the solutions provided by Open Data, Big Data and Artificial Intelligence among others. 


\section{Open Data}

Open Data is data freely available online for anyone to use and republish for any purpose. There are various sources of open data such as World Bank Open Data, World Health Organization - Open Data repository, Google Public Data Explorer, Data.gov, Registry of Open Data on AWS (RODA). It can help in a better understanding of the intercontinental issues, can aid businesses, can encourage machine learning and help fight rudimentary predicaments. It can also make the citizens aware about their rights, hence building a strong democracy. It can help create both social and economic value:

1. Promote wellbeing of population: Healthcare practitioners can make use of Open Data to make medical maintenances and other healthcare facilities more affordable and efficient.

2. Ensure availability of water and hygiene for all: Open Data in the form of images from various GPSs and satellites can be used to locate areas deficient in water supply and make amendments and an efficient scheme for placing water and sanitation points.

3. Establishing Global partnerships for sustainable development: Open Data can be also be used for global benefits. Various sovereign nations can come together and can implement a common solution to accomplish the aim of sustainable development that benefits everyone.

4. End poverty: Open Data helps in spreading the awareness about the subsidy provided by the government on the publicly available necessary goods and services.

5. Ensure comprehensive and unprejudiced education and aid long term learning opportunities for all: Numerous countries are now focusing on Open government data and other data to depict the availability and uplift the quality of schools.

\section{Big Data}

With the advances in ICT, the need of improving the capability, performance and precision of present-day data processing techniques is highly expanding. Big data is a developing concept enforced on data sets whose size is beyond the ability of commonly used computing platforms for capturing, managing and processing the data. Big data analytics in the sense of sustainable computing is a collection of unique ways that focus on new computing and system development issues in emerging sustainable applications. There are mainly 3 sources which generate big data, namely Social data, Transactional Data, and Machine data.[4]

Social Data: It comes from all the social media platforms like Twitter, Whatsapp, Instagram, Snapchat, Facebook, and others. All the chat histories, likes, comments, posts, shares, video uploads, and so on, are stored as big data. It gives a crucial perception of one's actions and emotions. It can be very dominating in marketing sector analysis. 
Machine Data: Various equipments, machine sensors, and user logs generate bulk of machine data. It is immensely increasing as the Internet of Things is expanding all over the world. It includes data from cameras, games, satellites, healthcare instruments, and other such data.

Transactional Data: It is the data that is produced by all the transactions be it online or offline. All the payment receipts, storage logs, invoices come under transactional data. All kinds of stores or websites such as of shopping, healthcare, stationery, and keep records of all the resources available, all the monetary matters, list of customers, price of each resource, etc.

Big data in sustainable computing can be used for humanitarian and developmental purposes [5]:

1. Epidemic prevention: Predicting the spread of various infectious diseases by mapping the movement of people with cell phones. One recent example is the detection of a fatal wide spreading disease caused by a virus called as Corona virus in China.

2. Effective monitoring of water quality: Water pump sensors placed can be used to detect the flow and access to clear and hygienic water.

3. Traffic monitoring: GPS devices can provide data that can be used to enhance the traffic control and to advance and promote the use of public transport.

4. Development of planned cities: Public land or spaces such as parks and streets can be monitored and encroachment can be tracked through satellite remote sensors.

5. Combating Global warming: Issues like deforestation can be avoided by combining merging satellite images, crowd-sourced witness accounts and open data.

6. Governance feedback: The use of Social media is increasing exponentially. People can provide their opinion and reviews about government policies building an improved world to live in. According to studies almost $80 \%$ of U.S. population uses social media. In India about 500 million people use social media.

\section{Artificial Intelligence}

Artificial Intelligence (AI) is defined as human intelligence stimulation in machines. In this, the machines are instructed to process and act like humans. The main purpose of AI is to compute and formulate a rational, logical and efficient way to accomplish a given task. In simple words, AI program machines to analyze a particular situation and take actions accordingly just like a human mind would.

There is real evidence to prove that AI has caused a major accession in world's development as well as in the sector of global economy. We make use of Artificial Intelligence in our everyday life. The analysis below in Figure 1, states the reference made to AI in corporate sustainability reports and CDP filings [6]. 


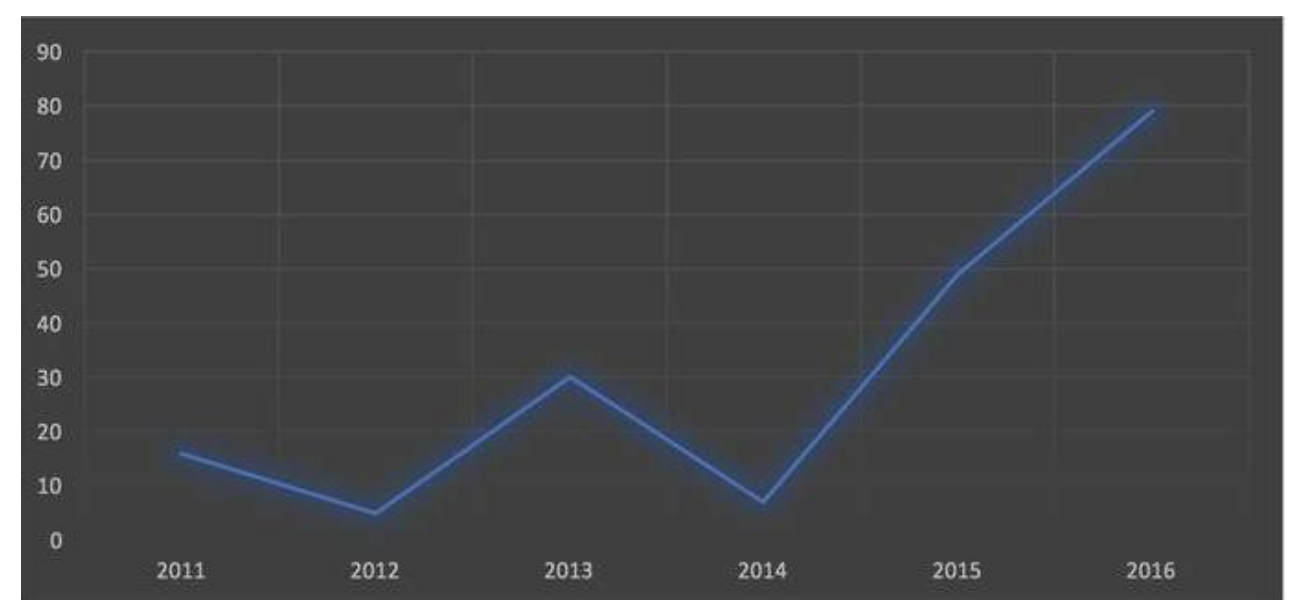

Fig. 1. References to AI in corporate sustainability reports and CDP filings

(Source: ESG Trends : esgtrends.com) [6]

AI has largely impacted the corporate world too. Companies have started using AI to achieve sustainability in terms of efficiency, emission prevention, and innovation of new technologies to provide varying services. Consider Xcel Energy, for example, it generates electricity by burning coal. A major greenhouse gas known as nitrous oxide $\left(\mathrm{N}_{2} \mathrm{O}\right)$ is emitted as a byproduct. This greenhouse gas causes climate change also causing depletion of ozone layer. Through AI this emission has been reduced to a great extent. The two plants of Xcel Energy in Texas have smokestacks which are now equipped with neural network. It is advance AI technology that stimulates a human brain. It analyses the data which is produced as a result from the combustion and provides the most efficient way to adjust the plant so as to minimize the emission of $\mathrm{N}_{2} \mathrm{O}$. Like Xcel Energy, AI has helped hundreds of companies around the world in reducing their $\mathrm{N}_{2} \mathrm{O}$ emissions.

Autonomous vehicles are one of the best known examples of AI. Presently one quarter of greenhouse gas comes from transport emissions. Self driving cars can provide a promising sustainable future. They could stand just inches between one another, improving efficiency and leaving more space on the road for cyclists or pedestrians.

Another mostly used feature is face detection. Mostly all the android or iOS phones use face recognition as security in their phones. It uses image processing feature of fizzy logic. They use of an algorithm that make use of a classifier called the fuzzy classifier in combination with HSV (Hue Saturation and Value) color model.[7] At first, the face is detected from a small window from the picture. Then HSV model comes into play, and helps in detection and extraction of the skin color. Even the smallest of things use AI.

AI has made the society we live in more efficient. It has immensely reduced the mistakes or errors humans make in calculations as it has a set of rules containing algorithms that it follows and hence the change of achieving the result correct if high. Today every home appliance from 
digital assistants like Alexa (form Amazon), Siri (from Apple), Natasha (from Hike), to refrigerators, air conditioners, or photocopy machines, everything uses AI by making use of its various technologies like Fuzzy logic, Genetic Algorithm, Neural Networks or mostly a combination of these technologies. It has also decreased the need to repeat the same task over and over again. Machines can now be adjusted to do the same job repetitively according to the conditions. All these benefits of AI have saved a massive amount of time.

To observe the affect of AI on sustainability, a sustainability analysis could be performed. The impact of AI could be examined on 5 different levels: individual, social, economical, technical, and environmental.

1. For an individual level, AI can strengthen learning, induce privacy concerns, impact work efficiency, affect health, and influence social interactions or can cause isolation.

2. On a social level, it can assist in organizations about legal frameworks, fraud detection, administering social media platforms, influencing connections or interactions, etc.

3. AI has predominantly affected the growth of globalization and the structures of organizations. On the other hand, it has grandly increased the rise in unemployment and may introduce many fiscal challenges. Hence affecting sustainability on an economic level.

4. At a technical level, AI may code for AI i.e. self learning algorithms might be extremely helpful. It might also help in understanding deep learning, engineering ethics, facial recognition, speech recognition, and so on.

5. Finally, AI, if put in good use may reduce pollution, help in waste management, spreading awareness of the need of sustainability, but if not used correctly can lead to magnify issues of resource and energy consumption. 


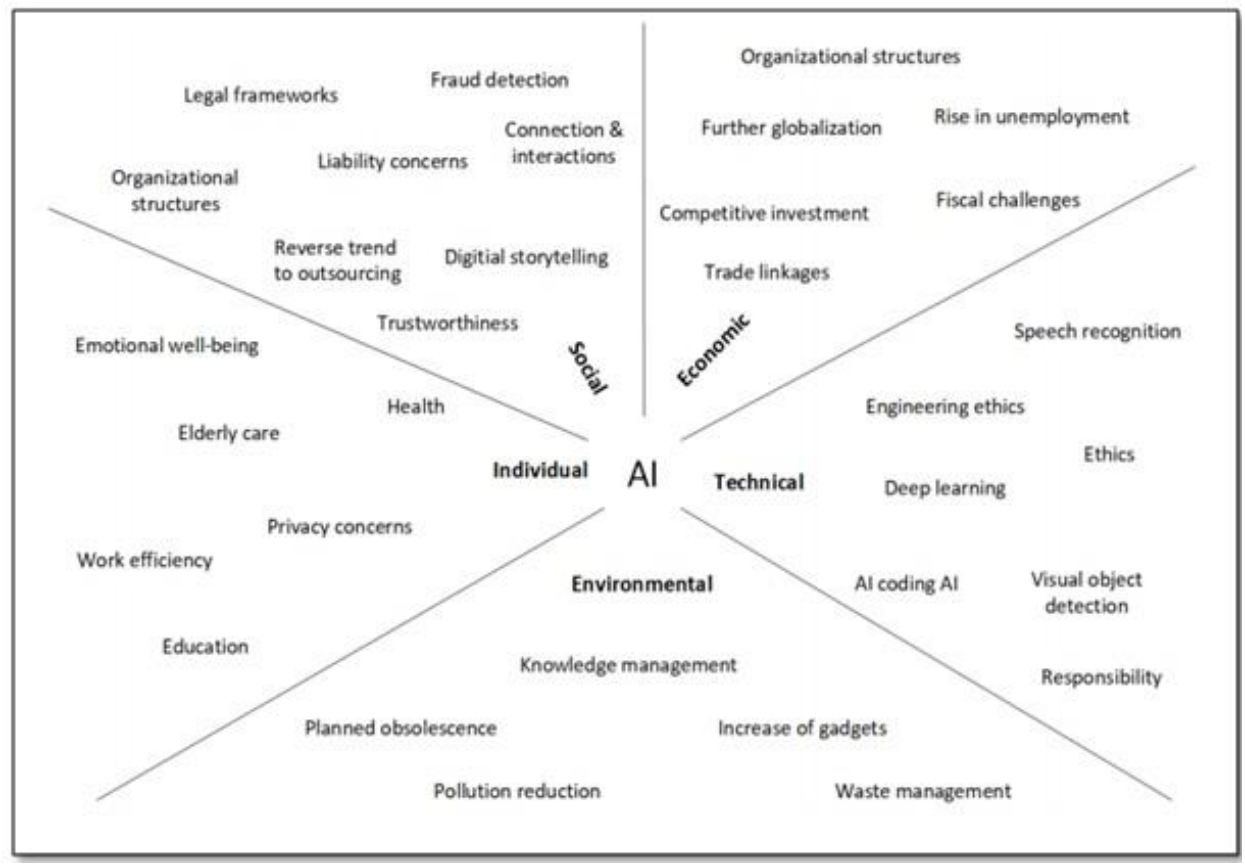

Fig. 2. Sustainability analysis diagram of Artificial Intelligence(AI) field according to the five categories.

(Source: The Rise of Artificial Intelligence under the Lens of Sustainability: Jayden Khakurel, Birgit Penzenstadler, Jari Porras, Antti Knutas and Wenlu Zhang [8])

Apart from all the perks, AI has some disadvantages too. Firstly, the cost of every complex machine has high maintenance and costly. They need regular cleaning or upgrades. If an unknown situation comes, the machines are not able think and make a decision, if no mention of such situation is in their algorithm. Machine also cannot be creative or invent a new idea. The human capability of originality thus remains unique. In today's world, people are using computers and technologies in a non ethical way by hacking into systems and invading privacy of any individual. Many financial frauds are committed during money transactions or from fake calls. Also the circulation of fake news is a big problem. Apart from these, it has a much higher impact on the employment. Nowadays machines are replacing humans, as machines can do some jobs better than humans in terms of time and accuracy.

Like every coin has 2 sides, AI also has a positive effect as well as a negative one. It depends on the developers to decide ethically as to what will help in building a sustainable future.

\section{Conclusion:}

This study demonstrates the need of Sustainable Computing and its impact on society. It is clear that the rapid growth of ICT industries globally is poisoning the environment. We must become more supporting towards the environmental issues and be energy conscious. Both, the 
government and the corporate world need to join hands in generating different sustainable computing solutions to be able to build a much better and secure sustainable future. Open data, big data and artificial intelligence are steps in achieving sustainable computing and for the world to have a promising future.

\section{References}

[1] Vinay Shukla, Dharmendra Singh, Shrawan Kumar, Rahul Singh: Sustainable Computing - A New Pathway to Go with Green (February 2015)

[2] P. K. Gupta, Mayank Singh: Sustainable Computing: A beginning (August 2016)

[3] Peter Corcoran: Cloud Computing and Consumer Electronics. A Perfect Match or a Hidden Storm? (April 2012)

[4] CloudMoyo: Sources of big data: Where does it come from? (2020)

[5] UN Global Pulse: Big Data for Sustainable Development (2017)

[6] Conor Riffle: What artificial intelligence means for sustainability (Wednesday, July 19, 2017)

[7] Akhay Bhatia, Smriti Srivastav, Ankit Agarwal: Face Detection Using Fuzzy Logic and Skin Color Segmentation in Images (2010)

[8] Jayden Khakurel, Birgit Penzenstadler, Jari Porras, Antti Knutas and Wenlu Zhang: The Rise of Artificial Intelligence under the Lens of Sustainability ( 3 November, 2018) 